\title{
Zum Inhalt von Band III
}

Im vorliegenden dritten Band „Elektrizität, Magnetismus, Elektromagnetische Schwingungen und Wellen“ werden die Grundlagen der Elektrostatik, elektrischer Ströme und magnetischer Felder besprochen. Die relativistische Betrachtung zeigt, dass ruhende Ladungen das elektrische Feld erzeugen, Magnetfelder aber mit bewegten Ladungen verknüpft sind. Alle elektromagnetischen Erscheinungen können in den Maxwell-Gleichungen zusammengefasst werden. Wichtig für die Anwendung sind der Wechselstromkreis und die Wirkung der Wechselstromwiderstände. Dies führt zum elektromagnetischen Schwingkreis und der Abstrahlung elektromagnetischer Wellen durch beschleunigte Ladungen, z. B. in einem Hertzschen Dipol. Elektromagnetische Wellen sind mit Energie- und Impulstransport und einem Drehimpuls verbunden. 
